\title{
Booms, busts and heavy-tails: the story of Bitcoin and cryptocurrency markets?
}

\author{
John Fry*
}

July 2018

\begin{abstract}
We develop bespoke rational bubble models for Bitcoin and cryptocurrencies that incorporate both heavy tails and the probability of a complete collapse in asset prices. Empirically, we present robustified evidence of bubbles in Bitcoin and Ethereum. Theoretically, we show that liquidity risks may generate heavy-tails in Bitcoin and cryptocurrency markets. Even in the absence of bubbles dramatic booms and busts can occur. We thus sound a timely note of caution.
\end{abstract}

Keywords: Bitcoin; Bubbles; Cryptocurrencies; Econophysics; Liquidity risk JEL Classification: C1 E4 G1

\section{Introduction}

Bitcoin and cryptocurrencies are the subject of much recent popular (Vigna and Casey, 2015) and academic (Gandal et al., 2018) interest. Economically, Bitcoin is interesting due to a lack of clarity over its definition (Fry and Cheah, 2016) with much recent work tending to classify Bitcoin as more of a speculative asset than a genuine currency (Baeck and Elbeck, 2015). We thus contribute to recent debates (see e.g. Cheah and Fry, 2015; Corbet et al., 2017) by developing a bespoke model for bubbles in cryptocurrency markets. In particular, it is the thesis of this paper that liquidity risks can generate heavy-tails in cryptocurrency prices even in the absence of speculative bubbles. Further, our model allows for the possibility that a lack of economic clarity may cause cryptocurrency prices to collapse completely (White, 2014).

Following previous work on liquidity (Tsuji, 2003) suppose that the returns $r_{t}$ of an asset are given by

$$
r_{t}=\lambda_{t} \ln Q_{t},
$$

*School of Computing Mathematics and Digital Technology, Manchester Metropolitan University, John Dalton Building, Chester Street, Manchester M1 5GD, UK.

E-mail: J.Fry@mmu.ac.uk 
where $Q_{t}$ is the trading volume and $\lambda_{t}$ is the liquidity. As a simplifying assumption suppose that $\lambda_{t}$ and $Q_{t}$ are independent and that $Q_{t}$ is log-normally distributed. If $\lambda_{t}$ is constant then asset returns are log-normally distributed in agreement with classical models (Black and Scholes, 1973). If $\lambda_{t}$ is stochastic then equation (1) leads to heavy-tailed asset returns related to market illiquidity. If, as an extreme example, the expected liquidity does not exist e.g.

$$
\frac{1}{\lambda_{t}} \sim N\left(0, \sigma_{1}^{2}\right),
$$

then the asset returns are Cauchy distributed. Taken together equations (1-2) give a plausible mechanism through which liquidity risks can generate heavy-tails in cryptocurrency prices. A simulated sample path from the Cauchy random walk model shown in Figure 1 demonstrates that in this case the nature of the market means that dramatic boom-bust episodes can occur even without the destabilising effect of a speculative bubble. We thus develop new models for speculative bubbles in an environment where the underlying level of financial risk is extreme.

[Insert Figure 1 about here]

The layout of this paper is as follows. Section 2 introduces the model used. Empirical applications to Bitcoin and other cryptocurrency markets are discussed in Section 3. Section 4 concludes.

\section{The model}

Let $P_{t}$ denote the price of a cryptocurrency at time $t$. The set up of the model is as follows. Adapting the original model in Johansen et al. (2000) our starting point is the equation

$$
P(t)=e^{X_{-}(t)}\left[1-H\left(t-t_{0}\right)\right],
$$

where $t_{0}$ denotes the time of the crash and $H(\cdot)$ denotes the Heaviside function. When a crash occurs the asset price collapses completely. This follows qualitative features of past cryptocurrency crashes (White, 2014). The timing of the crash $t_{0}$ is assumed to be unknown but described by the probability density $f(t)$ and CDF $F(t)$. Further, $X_{-}(t)$ satisfies the stochastic differential equation

$$
d X_{-}(t)=\mu(t) d t+\sigma(t) d W_{-}(t)
$$

where $W_{-}(t)$ is symmetric Cauchy noise (Samarodnitskiy and Taqqu, 1994). Set up in this way the model also incorporates empirical evidence of extreme heavy-tails in empirical cryptocurrency prices (Gkillas and Katsiampa, 2018). Use of the Cauchy distribution as a financial model (Harris, 2017) is also motivated by analytical tractability (Samarodnitskiy and Taqqu, 1994), 
classical financial models (Mandelbrot, 1963) and the study of Black Swans and heavy-tailed phenomena (Taleb, 2007). The Cauchy parameters $\mu$ and $2 \sigma$ also have a convenient interpretation as the median and the inter-quartile range respectively (Lee, 1997). These are often thought to be more robust summaries of financial risk and return (McNeil et al., 2005) alongside further links to theoretically coherent measures of financial risk (Artzner et al., 1999).

Taking logarithms it follows that prior to the crash $X_{-}(t)=\log P(t)$ satisfies the equation

$$
d X_{-}(t)=\mu(t) d t+\sigma(t) d W_{-}(t)-\frac{\delta\left(t-t_{0}\right)}{1-H\left(t-t_{0}\right)}
$$

Modifying the risk and return definitions in a related model in Cheah and Fry (2015) we have that:

Assumption 1 (Intrinsic Rate of Return) The intrinsic rate of return is assumed to be asymptotically constant:

$$
\operatorname{Median}\left[X_{t+\Delta}-X_{t} \mid X_{t}\right]=\mu(t) \Delta+o\left(\Delta^{2}\right) .
$$

Assumption 2 (Intrinsic Level of Risk) The intrinsic level of risk is assumed to be asymptotically constant:

$$
\text { Inter Quartile Range }\left[X_{t+\Delta}-X_{t} \mid X_{t}\right]=2 \sigma(t) \Delta+o\left(\Delta^{2}\right) \text {. }
$$

Suppose that a crash has not occurred by time $t$. The median return is given by

$$
\int_{t}^{t+\Delta} \mu(u) d u+\left[\int_{t}^{t+\Delta} \sigma(u) d u\right] \tan \left[\frac{\pi}{2}\left(\frac{q}{q-1}\right)\right]
$$

where $q=\frac{F(t+\Delta)-F(t)}{1-F(t)}=\Delta h(t)+o(\Delta)$ is the probability that a crash occurs during time $[t, t+\Delta]$ and $h(t)$ is the hazard rate. It follows from Assumption 1 and equation (8) that

$$
\begin{gathered}
\Delta \mu(t)+\frac{\mu^{\prime}(t) \Delta^{2}}{2}+o(\Delta)+[\Delta \sigma(t)+o(\Delta)]\left[-\frac{\pi}{2} \Delta h(t)+o(\Delta)\right]=\Delta \mu(t)+o\left(\Delta^{2}\right), \\
\mu^{\prime}(t)=\pi h(t) \sigma(t) .
\end{gathered}
$$

Relatedly, the inter-quartile range of the return is given by

$$
\left[\int_{t}^{t+\Delta} \sigma(u) d u\right]\left[\tan \left(\frac{\pi}{2}-\frac{3 \pi}{4(1-q)}\right)-\tan \left(\frac{\pi}{2}-\frac{\pi}{4(1-q)}\right)\right] .
$$


Expanding (10) in a power series gives

$$
\left[\Delta \sigma(t)+\frac{\Delta^{2} \sigma^{\prime}(t)}{2}+o(\Delta)\right]\left[2+\frac{\Delta \pi h(t)}{4}+o(\Delta)\right]=2 \sigma(t) \Delta+o(\Delta) .
$$

Thus equation (11) gives

$$
\sigma^{\prime}(t)+\frac{\pi h(t) \sigma(t)}{4}=0 ; \sigma(t)=\sigma(1-F(t))^{\frac{\pi}{4}}
$$

From equations (12) and (9) it follows that

$$
\mu(t)=\mu+4 \sigma-4 \sigma(1-F(t))^{\frac{\pi}{4}} .
$$

In the absence of a bubble $1-F(t)=1$ and it follows from equations (12) and (13) that in this case $\mu(t)=\mu, \sigma(t)=\sigma$ and the model reduces to a Cauchy distributed random walk. More generally, if $F(t) \neq 0$, it follows from equations (12-13) that the full solution of the model is

$$
X_{-}(t)=X_{0}+(\mu+4 \sigma) t-4 \sigma \int_{0}^{t}(1-F(u))^{\frac{\pi}{4}} d u+\sigma \int_{0}^{t}(1-F(u))^{\frac{\pi}{4}} d W_{-}(u)
$$

Following a related model in Zeira (1999) we assume that $t_{0} \sim U(0, \bar{T})$. It follows that in this case prior to the crash the log-returns $\Delta X_{t}=X_{t+1}-X_{t}$ are Cauchy distributed with parameters*

$$
\begin{aligned}
\text { location } & =(\mu+4 \sigma)+\frac{16 \sigma \bar{T}^{-\frac{\pi}{4}}}{\pi+4}\left[(\bar{T}-t-1)^{1+\frac{\pi}{4}}-(\bar{T}-t)^{1+\frac{\pi}{4}}\right] \\
\text { scale } & =\frac{4 \sigma \bar{T}^{-\frac{\pi}{4}}}{4+\pi}\left[(\bar{T}-t)^{1+\frac{\pi}{4}}-(\bar{T}-t-1)^{1+\frac{\pi}{4}}\right]
\end{aligned}
$$

Statistical tests for bubbles. From the above it follows that we can test for the presence of a speculative bubble by testing the null hypothesis $H_{0}: 1 / \bar{T}=0(F(t)=0)$ against the alternative hypothesis $H_{1}: 1 / \bar{T} \neq 0(F(t) \neq 0)$. As an illustration a Monte Carlo simulation of the likelihood ratio statistic under the null hypothesis of no speculative bubble in Bitcoin is shown in Table 1 and in Figure 2 and shows a reasonable agreement with a non-standard 50:50 mixture of a $\chi_{0}^{2}$ and $\chi_{1}^{2}$ distribution discussed in Self and Liang (1987) though results suggest that in practice this asymptotic approximation may be conservative.

\section{[Insert Table 1 and Figure 2 about here]}

Economic size of the effect. Robustified estimates of the economic size of the effect can be obtained in terms of the median price $\widetilde{P}(t)$. Refining a previous approach in Cheah and Fry

\footnotetext{
${ }^{*}$ A random variable $X$ has a Cauchy distribution with location parameter $\mu$ and scale parameter $\sigma$ if the probability density function of $X$ is given by $f(x)=\frac{\sigma}{\pi\left((x-\mu)^{2}\right)+\sigma^{2}}$, see e.g. Samorodnitskiy and Taqqu (1994), Ch. 1.
} 
(2015) define the fundamental value as the median price when a bubble is absent:

$$
P_{F}(t):=[\widetilde{P}(t)]=P_{0} e^{\mu t}
$$

In the presence of a bubble define

$$
P_{B}(t):=[\widetilde{P}(t)]=P_{F}(t) \exp \left\{4 \sigma t-\frac{16 \sigma \bar{T}}{\pi+4}\left[1-\left(1-\frac{t}{\bar{T}}\right)^{1+\frac{\pi}{4}}\right]\right\} .
$$

An estimated bubble component can then be defined in terms of the average distance between fundamental and bubble prices:

$$
\begin{aligned}
\text { Bubble Component } & =1-\frac{1}{T} \int_{0}^{T} \frac{P_{F}(t)}{P_{B}(t)} d t \\
& =1-\frac{1}{T} \int_{0}^{T} \exp \left\{\frac{16 \sigma \bar{T}}{\pi+4}\left[1-\left(1-\frac{t}{\bar{T}}\right)^{1+\frac{\pi}{4}}\right]-4 \sigma t\right\} d t
\end{aligned}
$$

where $T$ represents the length of the estimation window.

\section{Empirical application}

In this section we fit our model to empirical prices from the four major cryptocurrencies as determined by their current market capitalisation. Motivated by the recent boom and bust in cryptocurrencies we closing prices for Bitcoin and Ripple from January 1st 2015-January 1st 2018 and compare against newer altcoins that have only come into being more recently. As such, we look at Ethereum prices from August 7th 2015-January 1st 2018 and Bitcoin Cash prices from July 23rd 2017 to January 1st 2018. In common with similar studies the data are obtained from the authoritative website coinmarketcap.com. A plot of the data is shown in Figure 3. Summary statistics are shown in Table 2 and present clear evidence of the heavy-tails and excess kurtosis that our model can account for.

\section{[Insert Table 2 and Figure 3 about here]}

Results of our model applied to this data are shown in Tables 3-4. Evidence of bubbles is found in Bitcoin and Ethereum and clearly constitutes a significant proportion of observed prices. In contrast, no evidence of a bubble is found in Ripple prices once we account for heavy-tails and background levels of liquidity risk. This lack of a bubble may reflect technical advantages of Ripple relative to Bitcoin (Swan, 2015). Relatedly, no conclusive evidence of a bubble is found in the price of Bitcoin Cash - despite signs of marked volatility. However, there is some uncertainty related to a reduced sample size and the relatively recent inception of Bitcoin cash. A plot of the Google Relative Search Trends Index is shown in Figure 4 and suggests that generally higher 
numbers of searches may be linked to increased speculation and Bitcoin and Ethereum during the period in question though the number of searches decreases as the bubble comes to an end. This lends further support to the idea that cryptocurrencies are inherently speculative in nature (Baeck and Elbeck, 2015). Figure 5 shows that plotting Google Relative Search Trends index for Bitcoin and Ripple over an extended time period leads to similar conclusions.

[Insert Tables 3-4 and Figures 4-5 about here]

\section{Conclusions and discussion}

The evolution of financial technology changes the nature of financial risk (Cliff and Northrup, 2012). Fears have been raised over excessive speculation and price manipulation in Bitcoin and cryptocurrency markets (Gandal et al., 2018). A lack of clear accountability, coupled with liquidity risks, means that these markets are inherently risky. Market participants should exercise caution. In this paper we develop a rational bubble model for cryptocurrencies that combines heavy-tails with more realistic measures of risk and return and incorporates the possibility that in the absence of central regulation cryptocurrencies can collapse completely. We find evidence of bubbles in Bitcoin and Ethereum. In contrast no evidence of a bubble is found in Ripple once we account for heavy-tails and liquidity risk. This represents a theoretical refinement of the model in Cheah and Fry (2015) which fits a general asset bubble model to cryptocurrency data without accounting for specialised features of these markets. From a practical perspective findings may reflect technical advantages of Ripple relative to Bitcoin (Swan, 2015) and reduced levels of speculation according to Google data. However, the risk-management implications remain unclear as bubbles are not a necessary pre-requisite for boom-bust episodes to occur.

\section{Acknowledgements}

The author would like to acknowledge helpful comments and suggestions from Matt Burke, Dean Fantazzini and an anonymous reviewer. The usual disclaimer applies.

\section{References}

[1] Artzner, P., Delbaen, F., Eber, J. M., Heath, D. 1999. Coherent measures of risk. Math. Financ, 9, 203-228.

[2] Baeck, C., Elbeck, M. 2015. Bitcoins as an investment or speculative vehicle? A first look. Appl, Econ. Lett. 22, 30-34.

[3] Black, F., Scholes, M. 1973. The pricing of options and corporate liabilities. J. of Polit. Econ. 72, 637-659. 
[4] Cheah, E-T., Fry, J. M. 2015. Speculative bubbles in Bitcoin markets? An empirical investigation into the fundamental value of Bitcoin. Econ. Lett. 130, 32-36.

[5] Cliff, D. Northrup, L. 2012. The global financial markets: an ultra-large-scale systems perspective, in: Calinescu, R., Garlan, D. (Eds.) Monterey workshop 2012, lncs 7539, pp. 29-70.

[6] Corbet, S., Lucey, B., Yarovya, L. 2017. Datestamping the Bitcoin and Ethereum bubbles. Financ. Res. Lett. (forthcoming)

[7] Fry, J., Cheah, E-T. 2016. Negative bubbles and shocks in cryptocurrency markets. Int. Rev. of Financ. Anal., 47, 343-352.

[8] Gandal, N., Harrick, J. T., Moore, T., Oberman, T. 2018. Price manipulation in the Bitcoin ecosystem. J. of Monetary Econ. 95, 86-96.

[9] Gkillas, K. and Katsiampa, P. 2018. An application of extreme value theory to cryptocurrencies. Econ. Lett. 164, 109-111.

[10] Harris, D. E. 2017. The distribution of returns. Journal of Mathematical Finance 7, 769-804.

[11] Johansen, A., Ledoit, O., Sornette, D., 2000. Crashes as critical points. Int. J. of Theor. and Appl. Financ. 3, 219-255.

[12] Lee, P. M. 1997. Bayesian statistics: an introduction, second ed. Arnold, London.

[13] Mandelbrot, B. 1963. The variation of certain speculative prices. J. of Bus. 36, 394-419.

[14] McNeil, A. J., Frey, R., Embrechts, P. 2005. Quantitative risk management. Princeton University Press, Princeton.

[15] Samorodnitsky, G., Taqqu, M. S. 1994. Stable non-Gaussian random processes: Stochastic models with infinite variance. Chapman and Hall/CRC, Boca Raton London New York

[16] Self, S. G., Liang, K-Y. 1987. Asymptotic properties of maximum likelihood estimators and likelihood ratio tests under nonstandard conditions. J. of the Am. Stat. Assoc. 82, 605-610.

[17] Swan, M. 2015. Blockchain: blueprint for a new economy. O’Reilly Media.

[18] Taleb, N. N. 2007. The Black Swan: the impact of the highly improbable. Random House.

[19] Tsuji, C. 2003. Is volatility the best predictor of market crashes? Asia-Pac. Financ. Market. 10, 163-185.

[20] Vigna, P., Casey, M. 2015. The age of cryptocurrency: How Bitcoin and digital currency are changing the global economic order. St Martin's Press, New York. 
[21] White, L. 2014. The market for cryptocurrencies. GMU Working Paper in Economics No. 14-45

[22] Zeira, J. 1999. Informational overshooting, booms and crashes. J. of Monetary Econ. 43, 237-257. 


\section{Figures and Tables}

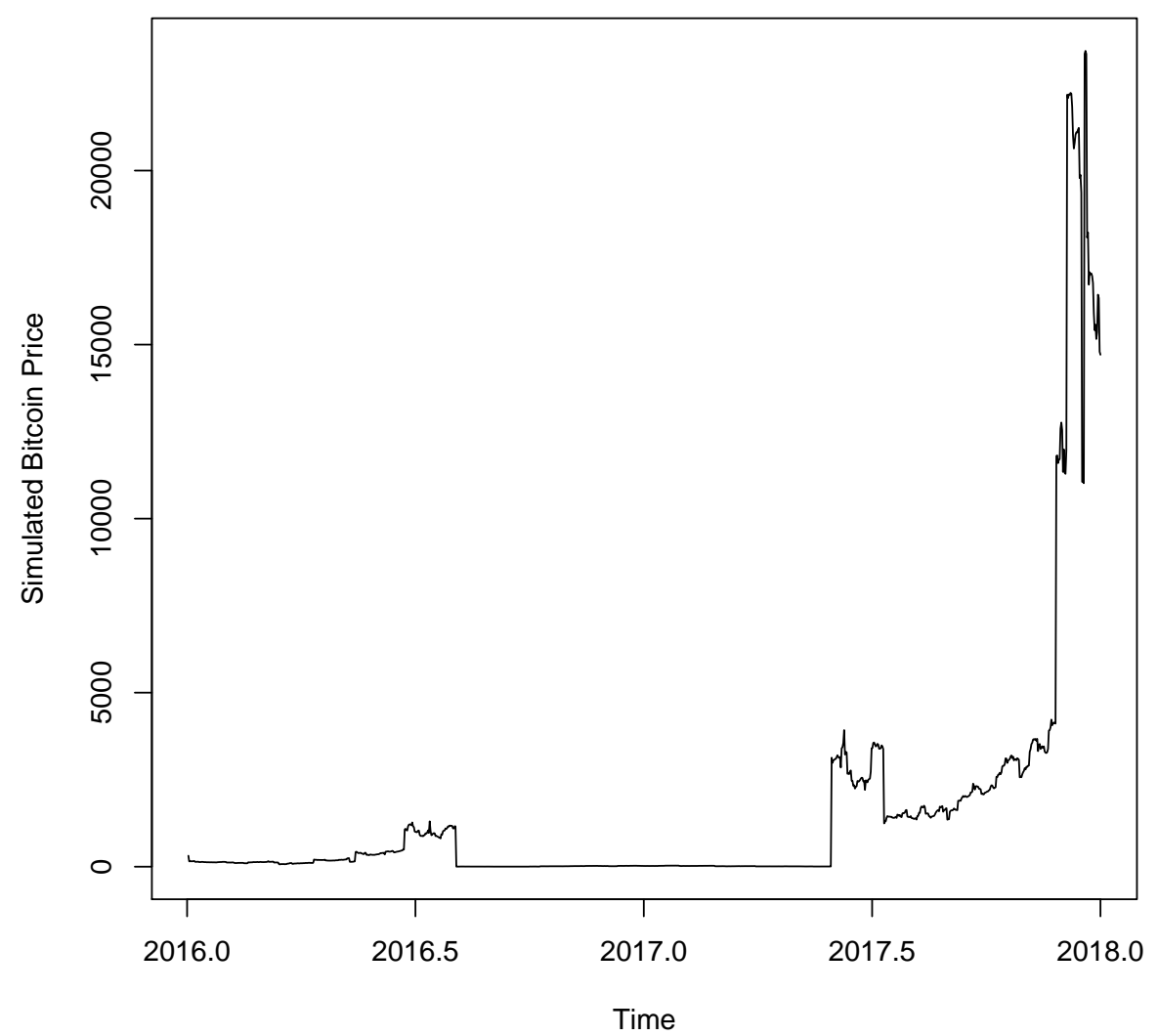

Figure 1: Example sample path of simulated Bitcoin prices under a Cauchy random walk assuming a Bitcoin price of 314.25 on January 1st 2015 and using parameters estimated by maximum likelihood.

\begin{tabular}{|l|l|l|}
\hline Statistic & Monte Carlo simulation & $\chi^{2}$ mixture distribution \\
\hline Mean & 0.410 & 0.5 \\
Median & 0 & 0 \\
$F^{-1}(0.9)$ & 1.320 & 1.642 \\
$F^{-1}(0.95)$ & 2.340 & 2.706 \\
$F^{-1}(0.99)$ & 4.932 & 5.412 \\
$F^{-1}(0.999)$ & 9.145 & 9.550 \\
\hline
\end{tabular}

Table 1: Comparison of Monte Carlo simulations with $\chi^{2}$ mixture distribution. 


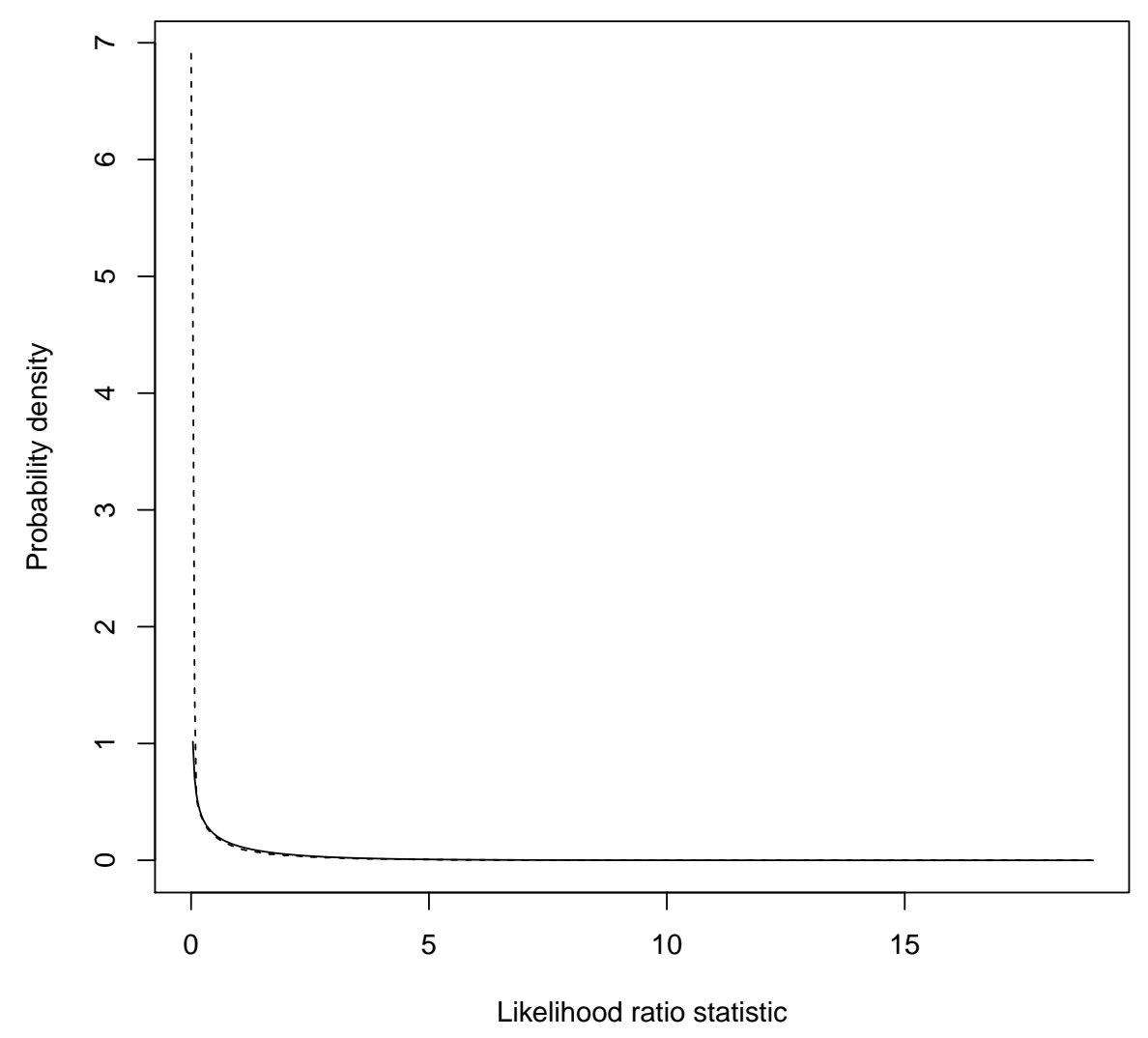

Figure 2: Distribution of the likelihood ratio statistic under the null hypothesis of no speculative bubble. Dashed line Monte Carlo simulation based on 100,000 simulations. Solid line, $\chi^{2}$ mixture distribution.

\begin{tabular}{|l|l|l|l|l|}
\hline Cryptocurrency & Bitcoin & Ethereum & Ripple & Bitcoin Cash \\
\hline Mean & 0.003441454 & 0.006413401 & 0.004183281 & 0.01094505 \\
\hline Median & 0.002545052 & -0.000437772 & -0.003363368 & -0.004980641 \\
\hline Maximum & 0.225119 & 0.4123373 & 1.027356 & 0.4315819 \\
\hline Minimum & -0.2375701 & -1.302106 & -0.6162727 & -0.4460382 \\
\hline St. Dev & 0.03844948 & 0.08519927 & 0.07305693 & 0.1247863 \\
\hline Skewness & -0.3492134 & -3.719406 & 3.802519 & 0.5728303 \\
\hline Kurtosis & 7.037797 & 64.47281 & 49.25887 & 2.599135 \\
\hline
\end{tabular}

Table 2: Summary statistics for log-returns from cryptocurrency markets. 

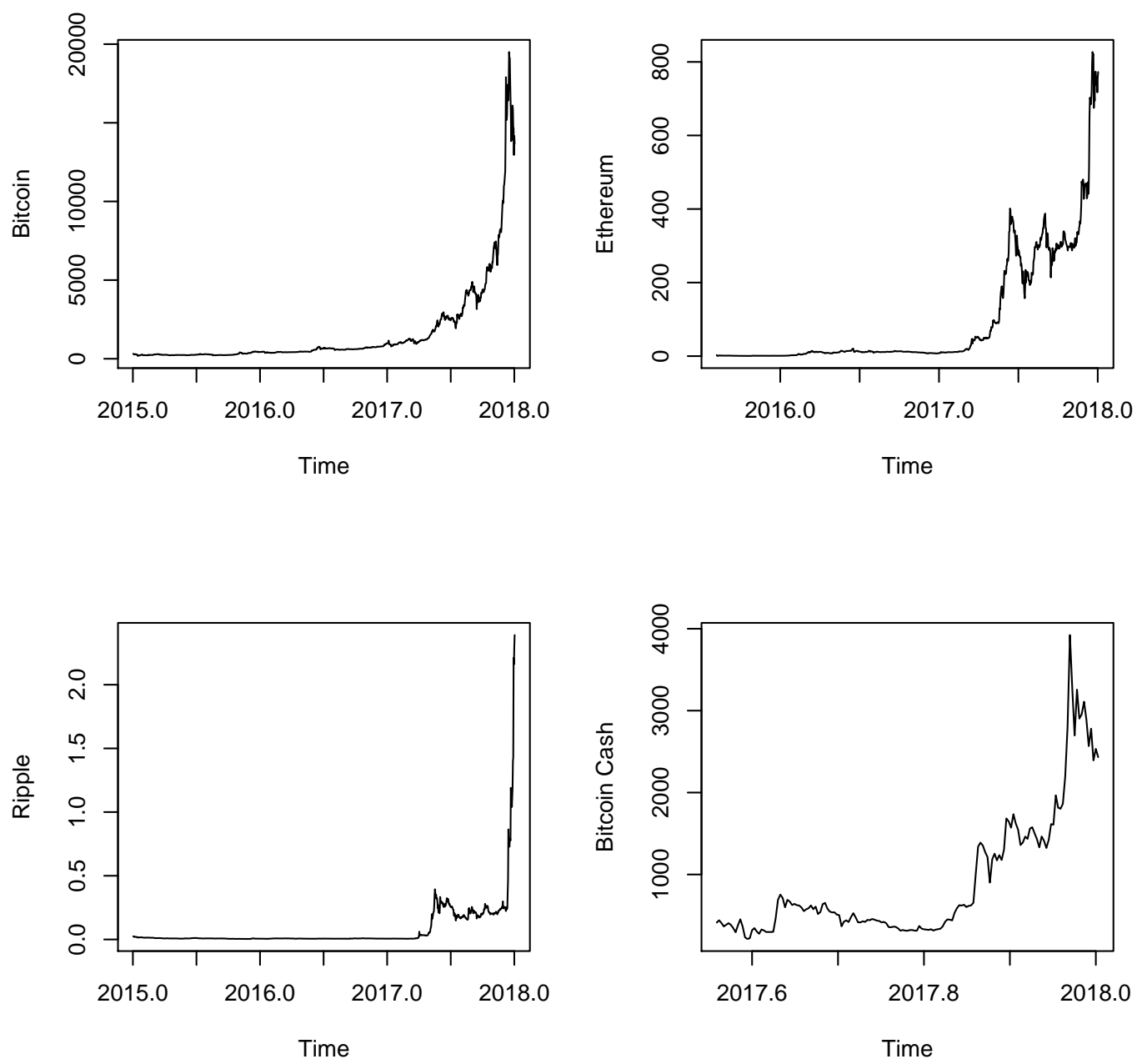

Figure 3: Plot of cryptocurrency prices over time: Bitcoin (top left), Ethereum (top right), Ripple (bottom left), Bitcoin Cash (bottom right).

\begin{tabular}{|l|l|l|}
\hline \multicolumn{3}{|l|}{$\begin{array}{l}\text { Maximum likelihood ratio test for a } \\
\text { speculative bubble } H_{0}: \frac{1}{\bar{T}}=0, H_{1}: \frac{1}{\bar{T}} \neq 0\end{array}$} \\
\hline Cryptocurrency & LR-test & $p$-value \\
\hline Bitcoin & 6.248 & 0.006 \\
Ethereum & 12.358 & 0.000 \\
Ripple & 0.000 & 0.500 \\
Bitcoin Cash & 0.6092 & 0.218 \\
\hline
\end{tabular}

Table 3: Results of speculative bubble (likelihood ratio) test. 


\begin{tabular}{|l|l|l|l|l|}
\hline Cryptocurrency & Estimate & $\begin{array}{l}\text { Estimated } \\
\text { Standard Error }\end{array}$ & $t$-value & $p$-value \\
\hline Bitcoin & 0.502 & 0.108 & 4.636 & 0.000 \\
Ethereum & 0.672 & 0.044 & 15.191 & 0.000 \\
Ripple & 0.000 & 0.000 & 0.017 & 0.493 \\
Bitcoin Cash & 0.375 & 0.266 & 1.410 & 0.079 \\
\hline
\end{tabular}

Table 4: Estimated bubble component shown in equation (17) and test of the null hypothesis bubble component $=0$.

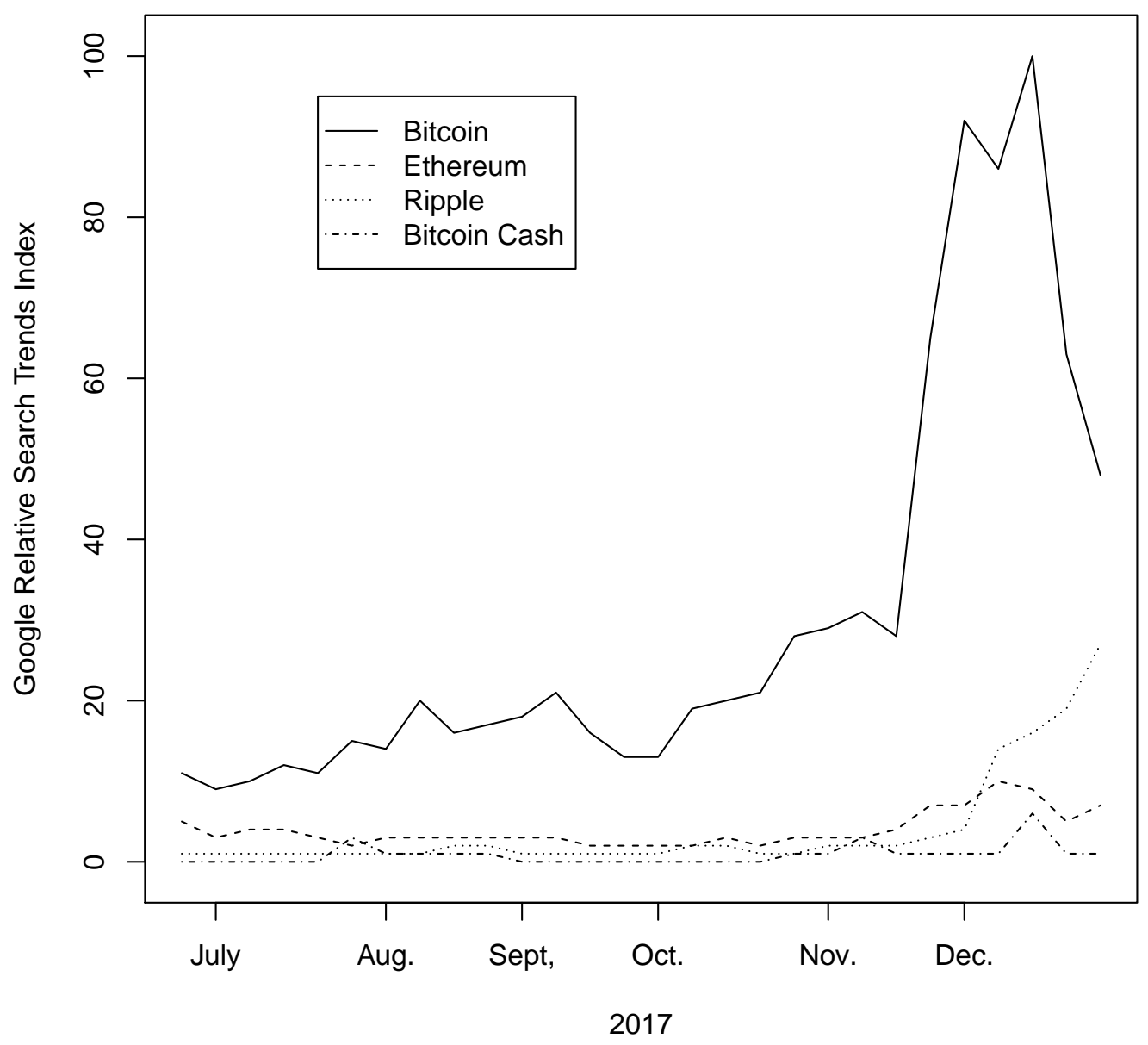

Figure 4: Plot of Google Relative Search Trends index over time: weekly data from 25/6/2017 to $31 / 12 / 2017$. 


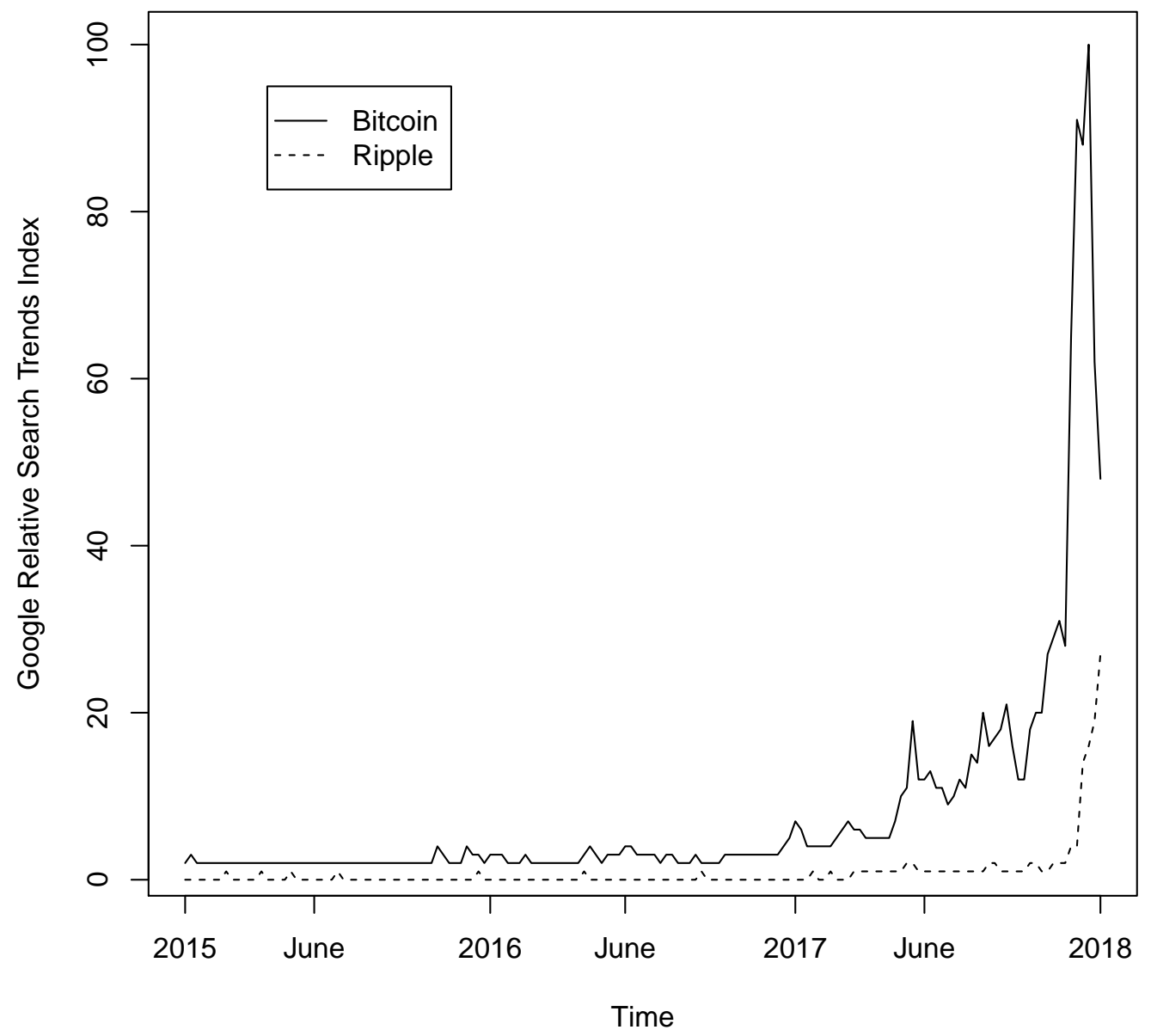

Figure 5: Plot of Google Relative Search Trends index over time: weekly data from 4/1/2015 to $31 / 12 / 2017$. 